\title{
The Inpatient Blindside: Comorbid Mental Health Conditions and Readmissions among Hospitalized Children
}

\author{
Jessica L. Bettenhausen, MD*, Katherine A. Auger, MD, MS², Jeffrey D. Colvin, MD, JD
}

'Department of Pediatrics, Children's Mercy Kansas City, University of Missouri-Kansas City School of Medicine, Kansas City, Missouri; ${ }^{2}$ Department of Pediatrics, University of Cincinnati, Cincinnati Children's Hospital Medical Center, Cincinnati, Ohio.

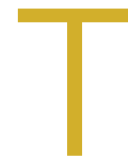

o ensure hospital quality, the Centers for Medicaid \& Medicare Services have tied payments to performance measures, including readmissions. ${ }^{1}$ One readmission metric, the Potentially Preventable Readmission measure (3M, PPR), was initially developed for Medicare and defined as readmissions related to an index admission, excluding those for treatment of cancer, related to trauma or burns, or following neonatal hospitalization. The PPR includes readmissions for both primary mental health conditions (MHCs) and for other hospitalizations with comorbid MHCs. ${ }^{2}$ Although controversies surround equating a hospital's quality with its rate of readmissions, the PPR has been expanded to include numerous states. Since the PPR is also used for the Medicaid population in these states, it also measures pediatric readmissions. Hospitals in states adopting PPR calculations, including children's hospitals, must either meet these new quality metrics or risk financial penalties. In light of evidence of high readmission rates among adult patients with $\mathrm{MHCs}$, several states have modified the PPR to exclude MHCs and claims for mental health services. ${ }^{3-9}$

In their study, "Mental Health Conditions and Unplanned Hospital Readmissions in Children," Doupnik et al. provided compelling evidence that MHCs in children (similar to adults) are closely associated with readmissions. ${ }^{10} \mathrm{MHCs}$ are possibly underappreciated risk factors for readmission penalties and therefore represent a necessary point for increased awareness. Doupnik et al. calculated 30-day unplanned hospital readmissions of children with versus without comorbid MHCs using another standard measure, the Pediatric All-Condition Readmission (PACR) measure. The PACR measure excludes index admissions with a $\mathrm{MHC}$ as primary diagnosis but includes children with comorbid MHCs.

Doupnik et al. used a nationally representative cohort of all index hospitalizations of children aged 3-21 years from the 2013 Nationwide Readmission Database that allowed for estimates of $\mathrm{MHC}$ prevalence in the study population. ${ }^{11} \mathrm{~A}$ comorbid $\mathrm{MHC}$ was identified in almost 1 in 5 medical admissions and 1 in 7 procedural admissions. Comorbid substance abuse

\footnotetext{
*Address for correspondence: Jessica L. Bettenhausen, MD, Children's Mercy Hospital, Adele Hall Campus, 2401 Gillham Road, Kansas City, MO 64108; Telephone: (816) 802-1493; Fax: (816) 302-3493; E-mail: jlbettenhausen@cmh.edu
}

Received: February 22, 2018; Accepted: February 23, 2018

๑ 2018 Society of Hospital Medicine DOI 10.12788/jhm.2975 was identified in $5.4 \%$ of medical admissions and $4.7 \%$ of procedure admissions, making this diagnosis the most frequently coded stand-alone MHC. The authors' findings are particularly noteworthy given that diagnosis of MHCs is highly dependent upon coding and is therefore almost certainly underreported. In pediatric inpatient populations, the true prevalence of comorbid MHCs is probably higher.

Doupnik et al. observed that comorbid MHCs are a significant risk factor for readmission. After adjustment for demographic, clinical, and hospital characteristics, children with MHCs presented a nearly $25 \%$ higher chance of readmission for both medical and procedural hospitalizations. Children admitted with medical conditions and multiple MHCs yielded odds of readmission $50 \%$ higher than that of children without MHCs. Overall, the presence of MHCs was associated with more than 2,500 medical and 200 procedure readmissions.

Previous studies in adult populations have also found that comorbid MHCs are an important risk factor for readmissions. ${ }^{12,13}$ Other research describes that children with MHCs have increased hospital resource use, including longer lengths of stay and higher hospitalization costs. ${ }^{14-17}$ Further, children with MHCs as a primary diagnosis are more prone to readmission, with readmission rates approaching those observed in children with medical complexity in some cases. ${ }^{18,19} \mathrm{MHCs}$ are common among hospitalized children and have become an increasingly present comorbidity in primary medical or surgical admissions. ${ }^{17}$

One particular strength of this study lies in its description of the relationship between comorbid (not primary) MHCs and readmission following medical or surgical procedures in hospitalized children. This relationship has been examined in adult inpatient populations but less so in pediatric inpatient populations. ${ }^{12,13}$ This study provides insights into the relationships between specific MHCs and unplanned readmissions for certain primary medical or surgical diagnoses, including those for attention deficit disorder and autism that are not well-recognized in adult populations.

High-quality inpatient pediatric practice depends not only upon recognition of concurrent MHCs during hospitalizations but also assurance of follow-up outside of such institutions. During the inpatient care of children, pediatric hospitalists often perform myopic inpatient care which fails to routinely address underlying MHCs. ${ }^{20}$ For example, among children who are admitted with primary medical or procedure diagnoses, it is possible, or perhaps likely, that providers give little attention to an underlying $\mathrm{MHC}$ outside of continuation of a current 
medication. Comorbid MHCs are not accounted for within readmission calculations that directly affect hospital reimbursement. This study suggests that comorbid MHCs in hospitalized children may worsen readmission penalty status. In this manner, comorbid MHCs may represent a hospital's blindside.

We agree with Doupnik et al. that an integrated approach with medical and mental health professionals may improve the care of children with MHCs in hospitalized settings. This improvement in care may eventually affect hospital-level national quality metrics, such as readmissions. The findings of Doupnik et al. also provide a strong argument that pediatric inpatient providers should consider mental health consultations for patients with frequent admissions associated with chronic conditions, as comorbid MHCs are associated with worsened disease states and account for a disproportionate share of admissions for children with chronic conditions. ${ }^{21,22}$ Recognition of comorbid MHCs may improve baseline chronic disease states for hospitalized children.

\section{References:}

1. Centers for Medicare \& Medicaid Services. Hospital Readmission Reduction Program. https://www.cms.gov/Medicare/Quality-Initiatives-Patient-Assessment-Instruments/Value-Based-Programs/HRRP/Hospital-Readmission-Reduction-Program.html. Published September 28, 2015. Accessed February 9, 2018.

2. 3M. Potentially Preventable Readmissions Classification System. http://multimedia.3m.com/mws/media/10426100/resources-and-references-his-2015. pdf. Accessed February 9, 2018.

3. Illinois Department of Family and Healthcare Services. Hospital Inpatient Potentially Preventable Readmissions Information and Reports. https://www. illinois.gov/hfs/MedicalProviders/hospitals/PPRReports/Pages/default.aspx. Accessed February 9, 2018.

4. New York State Department of Health. Potentially preventable hospital readmissions among medicaid recipients with mental health and/or substance abuse health conditions compared with all others: New York State, 2007 https://www.health.ny.gov/health_care/managed_care/reports/statistics_ data/3hospital_readmissions_mentahealth.pdf. Accessed February 9, 2018.

5. Texas Health and Human Services Commission. Potentially preventable read missions in Texas Medicaid and CHIP Programs, Fiscal Year 2013. https://hhs. texas.gov/reports/2016/08/potentially-preventable-readmissions-texas-medicaid-and-chip-programs-fiscal-year-2013. Accessed February 9, 2018.

6. Oklahoma Healthcare Association. Provider reimbursement notice. https:// www.okhca.org/providers.aspx?id=2538. Accessed February 9, 2018.

7. Washington State Hospital Association. Potentially preventable readmission (PPR) adjustments. http://www.wsha.org/articles/hca-implements-potentially-preventable-readmission-ppr-adjustments/. Accessed February 9, 2018.

8. State of Colorado. HOIP 30-day All cause readmission. https://www.colorado.gov/pacific/sites/default/files/2016\%20March\%20HQIP\%2030-day\%20 all-cause\%20readmission\%20measure.pdf. Accessed February 9, 2018.

9. Maryland Health Services Cost Review Commission. Readmission reduction incentive program. http://www.hscrc.state.md.us/Pages/init-readm-rip.aspx. Accessed February 9, 2018.

10. Doupnik SK, Lawlor J, Zima BT, et al. Mental health conditions and unplanned hospital readmissions in children. J Hosp Med. 2018(13):445-452.

11. NRD Overview. https://www.hcup-us.ahrq.gov/nrdoverview.jsp. Accessed February 9, 2018.
We assert that the current silos in inpatient pediatrics of medical and mental healthcare are outdated. Pediatric hospitalists need to assess for and access effective MHC treatment options in the inpatient setting. In addition to the provision of mental health care within hospital settings, providers should also ensure that appropriate follow-up is arranged at the time of discharge. From a health policy standpoint, providers should clarify how both primary and comorbid MHCs are included within readmission measures while considering the close association of these conditions with readmission. Although the care of children with MHCs requires a longterm and coordinated approach, identification and treatment during hospitalization offer unique opportunities to modify outcomes of MHCs and coexistent medical and surgical diagnoses.

Disclosures: The authors declare no conflict of interest.

12. Singh G, Zhang W, Kuo Y-F, Sharma G. Association of psychological disorders with 30-day readmission rates in patients with COPD. Chest. 2016;149(4):905915. doi:10.1378/chest.15-0449

13. Mclntyre LK, Arbabi S, Robinson EF, Maier RV. Analysis of risk factors for patient readmission 30 days following discharge from general surgery. JAMA Surg. 2016;151(9):855-861. doi:10.1001/jamasurg.2016.1258

14. Bardach NS, Coker TR, Zima BT, et al. Common and costly hospitalizations for pediatric mental health disorders. Pediatrics. 2014;133(4):602-609. doi:10.1542/peds.2013-3165

15. Doupnik SK, Lawlor J, Zima BT, et al. Mental health conditions and medical and surgical hospital utilization. Pediatrics. 2016;138(6): e20162416. doi:10.1542/peds.2016-2416

16. Doupnik SK, Mitra N, Feudtner C, Marcus SC. The influence of comorbid mood and anxiety disorders on outcomes of pediatric patients hospitalized for pneumonia. Hosp Pediatr. 2016;6(3):135-142. doi:10.1542/ hpeds.2015-0177

17. Zima BT, Rodean J, Hall M, Bardach NS, Coker TR, Berry JG. Psychiatric disorders and trends in resource use in pediatric hospitals. Pediatrics. 2016;138(5): e20160909. doi:10.1542/peds.2016-0909

18. Feng JY, Toomey SL, Zaslavsky AM, Nakamura MM, Schuster MA. Readmission after pediatric mental health admissions. Pediatrics. 2017;140(6):e20171571. doi:10.1542/peds.2017-1571

19. Cohen E, Berry JG, Camacho X, Anderson G, Wodchis W, Guttmann A. Patterns and costs of health care use of children with medical complexity. Pediatrics. 2012;130(6):e1463-e1470. doi:10.1542/peds.2012-0175

20. Doupnik SK, Walter JK. Collaboration is key to improving hospital care for patients with medical and psychiatric comorbidity. Hosp Pediatr. 2016;6(12):760-762. doi:10.1542/hpeds.2016-0165

21. Richardson LP, Russo JE, Lozano P, McCauley E, Katon W. The effect of comorbid anxiety and depressive disorders on health care utilization and costs among adolescents with asthma. Gen Hosp Psychiatry. 2008;30(5):398-406. doi:10.1016/j.genhosppsych.2008.06.004

22. Malik FS, Hall M, Mangione-Smith $R$, et al. Patient characteristics associated with differences in admission frequency for diabetic ketoacidosis in United States children's hospitals. J Pediatr. 2016;171:104-110. doi:10.1016/j. jpeds.2015.12.015 